\title{
A Simulation of Shell Structures of Comet Hale-Bopp in February 1997
}

\author{
J. Tao \& B.C. Qian \\ Shanghai Astronomical Observatory, Chinese Academy of Sciences, \\ Shanghai, 200030
}

\begin{abstract}
We present a simulation based on the morphology of the inner coma of comet Hale-Bopp(C/1995 O1) in February 1997. The synthetic images can fit the observations well, suggesting a simple spin mode. The rotation parameters, obliquity of orbital plane to equator and argument of subsolar meridian at perihelion, are 71 and 81 degrees respectively.
\end{abstract}

\section{Introduction}

Comet Hale-Bopp $(\mathrm{C} / 1995 \mathrm{O} 1)$ has been one of the active comets in recent years. The comet shows various coma morphology, such as fans and radial jets. Coma structures may be an indicator of the spin axis (Sekanina \& Larson 1984, 1986; Sekanina 1996, 1998a, 1998b; Vasundhara et al. 1999; Samarasinha et al. 19971999; Samarasinha 2000, Jorda et al. 1997-1999; Wang 1999). Most of these models have similar physical concepts: sources on the rotating nucleus are ejecting continuously from local sunrise to sunset. The basic concept of our model comes from Sekanina's work.

\section{Observations}

Optical observations of comet Hale-Bopp were carried out with the $1.56 \mathrm{~m}$ Telescope and a CCD camera (1024*1024pixels) at She-shan Station, Shanghai Astronomical Observatory. The camera offers a field of view of $13^{\prime *} 13^{\prime}$. The cousins filter I is used for direct imaging because this waveband is related to cometary dust structure. In Feb. 1997, we observed for 8 days and obtained CCD images of this comet. Each CCD image is corrected for bias, dark, and flat field. In order to depress the coma background and to clearly show the near nuclear structure, we processed the images with the wavelet method (Tao et al. 1997; Tao et al. 2000; Marchis et al. 1999). The coma structures can be clearly seen in Figure 1 (white patterns).

\section{Results and Discussion}

The obliquity of the orbit plane to the equator, is less than 90 degree for the prograde sense of rotation and ranges from 90 degree to 180 degree when rotation 
is retrograde relative to the comet's orbital motion. The phip is the argument of the subsolar meridian at perihelion. The coma morphology suggests the presence of two active regions. Three point sources for each region were used to simulate the coma patterns. The distance between each point source is 5 degree and these point sources are lined in the longitude direction.

By variation of the parameters listed in Table 1, we modeled the shell structure of the dust coma until the simulated result (black dots in Figure 1) provided optimum agreement with the observed shell features. Table 1 lists the results of spin orientation and position of the active sources. Our results are consistent with others (e.g. Sekanina 1998a,1998b; Jorda et al. 1997-1999; Vasundhara et al. 1999; Licandro et al. 1997-1999).

Table 1. Simulation result

\begin{tabular}{cccccc}
\hline \hline$\alpha$ & $\delta$ & Obliquity & phip & 1st latitude & 2nd latitude \\
\hline 250 & -60 & 71 & 81 & 30 & 0 \\
\hline
\end{tabular}

\section{References}

Jorda, L., Rembor, K., Lecacheux, J., et al. 1997-1999, Earth, Moon, and Planets, 77, 167

Licandro,J., Bellot Rubio, L.R., Casas, R., et al. 1997-1999, Earth, Moon, and Planets, 77,199

Marchis,F. et al. 1999, A\&A, 349, 985

Samarasinha, N. H. 2000, ApJ, 529, L107

Samarasinha, N.H., Mueller, B. E. A., Belton, M. J. S. 1997-1999, Earth, Moon, and Planets, 77, 189

Sekanina, Z. 1996, A\&A, 314, 957

Sekanina, Z. 1998a, ApJ, 494, L121

Sekanina, Z. 1998b, ApJ, 509, L133

Sekanina, Z \& Larson, S.M. 1984, AJ, 89, 1408

Sekanina, Z \& Larson, S.M. 1986, AJ, 92, 462

Tao, J., Qian, B., Tang.Y., et al. 1997, Progress in Astronomy, 15, 68

Tao, J., Qian, B., Gu, M., et al. 2000, Planetary and Space Science, 48, 153

Vasundhara, R.\& Chakraborty, P. 1999, Icarus, 140, 221

Wang, Y. 1999, Master dissertation, Nanjing University 


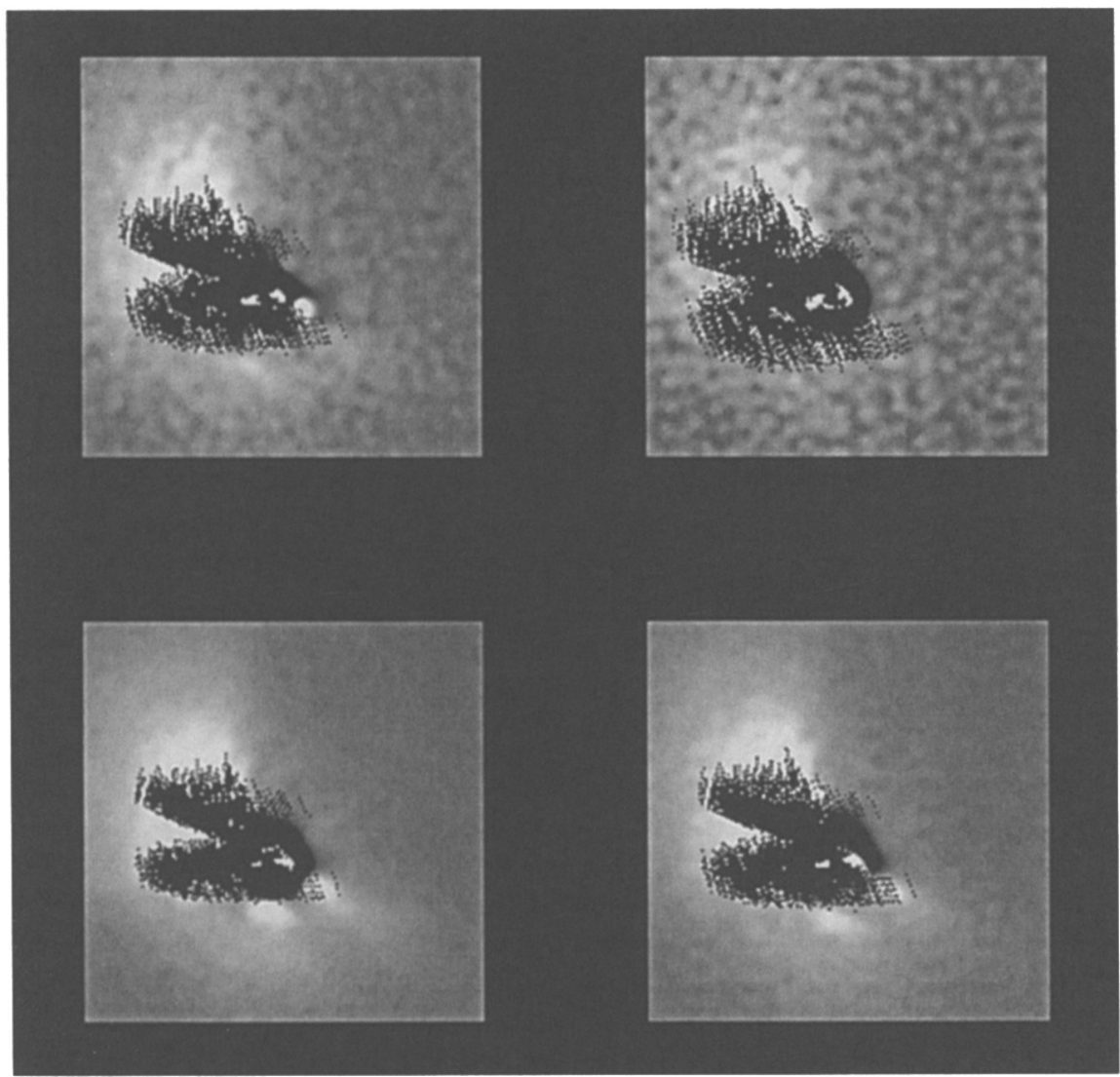

Figure 1. Observation images of Comet Hale-Bopp with synthetic features superposed. The observations were taken on Feb. 12 (upper left), Feb. 16 (upper right), Feb. 8 (lower left) and Feb. 10 (lower right) 1997. West is up and south to the left. The FOV is 2'.3 square size. An I filter is used. 

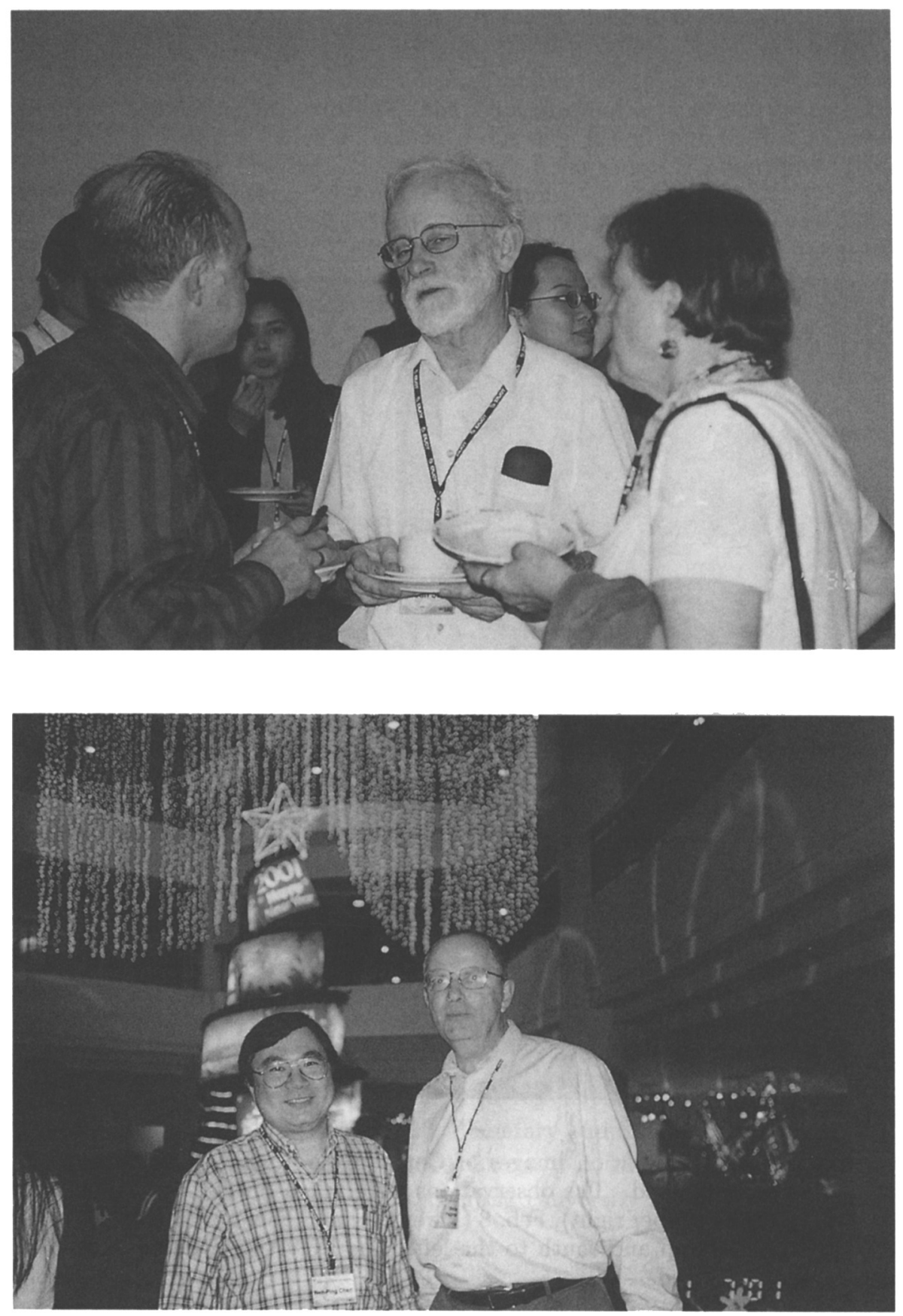

(Top, from left) Samus, C. Scarfe and A. Scarfe; (bottom, from left) Chen and Paczyński 